\title{
ON THE INVERSION OF THE CAUER-ROUTH MATRIX*
}

BY

W. H. INGRAM

Introduction. The Cauer-Routh matrix is defined and its application is described to the calculation of branch currents and node voltages in networks whose branches and nodes are so numerous that the inversion of the network impedance matrix is numerically intractable, to systems of networks interconnected by tie-lines, and in particular to cases where Bückner's formula can be applied with advantage.

1. Review of elementary network theory. Electrical network theory may be said to be the union, legitimized by Lagrange, of two lines of scientific thought. One began with Kirchhoff and has a line of development through von Helmholtz, Poincare and Veblen and the other may be said to have begun with $\mathrm{Ohm}$ and has a line of development through Faraday, Ampère and Maxwell. The first is characterized by its concern with the topology of electrical networks and the second by its concern with the laws of equilibrium of electromotive force, i.e., with the equation of a given driving e.m.f. to components which sustain current, charge and acceleration of current in any resistor, capacitor, or inductor, respectively, or combination of such in cyclic series with the source of said e.m.f. If we call any linear, as distinct from cyclic, series of one or more of these four elements a simple dipole then, when a set of simple dipoles is connected up to form a network, Lagrangian forces of the "first kind", i.e., forces of constraint, are thereby brought into existence and have to be taken into account in the e.m.f. equation. The Lagrangian formula for forces of the "second kind" is known to account for the mechanical forces and torques in any case where the inductances of network inductors are functions of position.

Let $\mathfrak{T}$ be a connected and nonseparable network ${ }^{1}$ of simple dipoles whose graph $\mathcal{G}$ consists of $b$ branches and $V$ vertices. The set of branch charges being represented by $Q$, branch currents by $\dot{Q}$, the electromotive forces by $E$ and the forces of constraint by $F$, the e.m.f. equation of the network is

$$
E(t)=F+[l] \ddot{Q}+[r] \dot{Q}+[s] Q .
$$

All the coefficients are assumed to be constants.

It is necessary to recall how the forces of constraint are expressed by means of the Veblen-Poincare directed-branch-on-vertex incidence matrix $\bar{\Pi}$ and the connected Lagrangian theory. First we construct a sagittal or directed graph $\overrightarrow{\mathcal{g}}$ by arbitrarily assigning direction to each branch to give a direction basis for $E$ and $\dot{Q}$. The equation $\bar{\Pi} \dot{Q}=0$ expresses the Kirchhoff condition at the $V$ vertices.

A most elementary theorem tells us that only $V-1$ rows of $\bar{I}$ are linearly independent. This being so, it is economical to call one vertex a ground point and the others, say $V_{1}, V_{2}, \cdots, V_{V-1}$, nodes. For the nodes, then, Kirchhoff's current condition may be

* Received April 25, 1968.

${ }^{1} \mathrm{~A}$ network is connected if there is a continuous path (possibly partly through the ground) from any first vertex in the graph of the network to any second and nonseparable if two such paths exist having no intermediate point in common. 
written

$$
\Pi \dot{Q}=0
$$

where II is $\bar{I}$ with the last row deleted.

By Lagrangian theory, a row-vector $\lambda$ of multipliers exists such that

$$
\lambda \Pi=F_{\mathbf{T}},
$$

and, because the elements of II are dimensionless, $\lambda$ is a set of $n$ forces, or potential differences, to wit: the set of node potentials $V_{1}, V_{2}, \cdots, V_{n}$ above the potential of the ground-point.

Let $i$ represent the set of root-mean-square values, element by element, of the periodic particular solution of " 1 " for $Q$, such periodic particular solutions being assumed to exist when $E(t)$ is a set of sinusoids with a common period. Let $e$ and $V$ have the same r.m.s. relation to $E(t)$ and $F$, respectively. Then instead of " 1 " and " 3 " we have

$$
\left[\begin{array}{cc}
Z & \Pi_{\mathrm{T}} \\
\Pi & 0
\end{array}\right]\left[\begin{array}{l}
i \\
V
\end{array}\right]=\left[\begin{array}{l}
e \\
0
\end{array}\right] .
$$

I call the matric coefficient here the Cauer-Routh matrix [1, p. 46], [2, p. 91]. As in classical dynamics, we assume that that the velocities (or analogue thereof) and internal stresses have functional dependence on the driving force $e$ and not, as here, the other way around: we assume that the Cauer-Routh matrix has an inverse and, moreover, that $Z$ has an inverse.

Let

$$
\begin{aligned}
{\left[\begin{array}{cc}
Z & \Pi_{\mathbf{T}} \\
\Pi & 0
\end{array}\right]^{-1}=\left[\begin{array}{cc}
A & B \\
C & -z
\end{array}\right] } \\
Z^{-1}=Y, \quad Y=\left[\Pi Y \Pi_{\mathbf{T}}\right]=z^{-1} .
\end{aligned}
$$

Obvious calculations give

$$
A=Y-Y \Pi_{\mathrm{T}} z \Pi Y, \quad B=Y \Pi_{\mathrm{T}} \mathfrak{z}, \quad C=z \Pi Y,
$$

and so

$$
i=A e, \quad V=C e .
$$

The number of nodes does not have to be very large for the inversion of $y$ to be very laborious to calculate numerically and the inversion of $Z$, since there are almost always more branches than nodes, may be expected to be even more laborious. The analysis of this case is the concern of the next section.

2. Networks interconnected by tie-lines. Suppose we have a number of networks quite isolated from each other except that some of the nodes of the $i$ th network are connected with some of the nodes of the $j$ th, possibly all $i \neq j$, by tie-lines which are dipoles having possibly mutual impedance with other tie-lines but not with any branch elsewhere in the system.

Let $Z$ be the branch-impedance matrix for the set of networks and $z$ the matric set of tie-line impedances. After directing the tie-lines arbitrarily, let $K$ represent the tieline-on-node incidence matrix. For currents $i^{\prime}$ in the branches of the set of networks and 
$i^{\prime \prime}$ in the tie-lines, co-directed with the branches in the sagittal graph of the system, Kirchhoff's node condition is

$$
\Pi i^{\prime}+K i^{\prime \prime}=0
$$

and the vector force of constraint is

$$
V_{\mathbf{T}}(\Pi, K)=F_{\mathbf{T}} .
$$

For co-directed e.m.f.s $e^{\prime}$ in the networks and $e^{\prime \prime}$ in the tie-lines, the equation of force is

$$
\left[\begin{array}{ccc}
Z & 0 & \mathrm{\Pi}_{\mathbf{T}} \\
0 & z & K_{\mathbf{T}} \\
\mathrm{II} & K & 0
\end{array}\right]\left[\begin{array}{c}
i^{\prime} \\
i^{\prime \prime} \\
V
\end{array}\right]=\left[\begin{array}{c}
e^{\prime} \\
e^{\prime \prime} \\
0
\end{array}\right]
$$

The matric coefficient here is the Cauer-Routh matrix with impedance matrix $[(Z, 0) /$ $(0, z)]$ and constraint matrix $(\Pi, K)$.

Let

$$
\left[\begin{array}{ccc}
Z & 0 & \mathrm{II}_{\mathbf{T}} \\
0 & z & K_{\mathbf{T}} \\
\mathrm{II} & K & 0
\end{array}\right]^{-1}=\left[\begin{array}{ccc}
A & B & C \\
D & E & F \\
G & H & -
\end{array}\right] ;
$$

then, because the product of the inverse of the first by the second is a partitioned identity matrix, we find that

$$
\begin{array}{lcc}
A=Y-Y \Pi_{\mathrm{T}} G, & B=-Y \Pi_{\mathrm{T}} H, & C=Y \Pi_{\mathrm{T}} z, \\
D=-y K_{\mathrm{T}} G, & E=y-y K_{\mathrm{T}} H, & F=y K_{\mathrm{T}} z, \\
G=z \Pi Y, & H=z K y, & z=\mathcal{Y}^{-1}, \\
y=\left[\Pi Y \Pi_{\mathrm{T}}+K y K_{\mathrm{T}}\right] . &
\end{array}
$$

The set of branch and tie-line currents and the set of node potentials thus are

$$
\begin{aligned}
i^{\prime} & =\left[Y-Y \Pi_{\mathrm{T}} z \Pi Y\right] e^{\prime}-Y \Pi_{\mathrm{T}} z K y e^{\prime \prime}, \\
i^{\prime \prime} & =-y K_{\mathrm{T}} z \Pi Y e^{\prime}+\left[y-y K_{\mathrm{T}} z K y\right] e^{\prime \prime}, \\
V & =z \Pi Y e^{\prime}+z K y e^{\prime \prime} .
\end{aligned}
$$

We have exchanged the generality of Sec. 1 for this particularity by imposing zero mutual inductance between the tie-lines and the branches of the networks which they connect. We consider now the case where the total number of branches and nodes is so great that the inversion of $Z$ and of $\Pi Y \Pi_{\mathrm{T}}$, required above, are intractable numerically. We cope with this situation by imposing zero mutual inductance between any one network and another and we number the branches of the first network first, the branches of the second network second and so forth and get $Z$ diagonalized in blocks. To correspondingly diagonalize $\Pi Y \Pi_{\mathrm{T}}$, we number the vertices in the same way. But the $i$ th block of $\Pi Y \Pi_{\mathrm{T}}, \Pi_{i} Y_{i} \Pi_{i \mathrm{~T}}$, is not invertible unless the rows of $\Pi_{i}$ are linearly independent and so it is necessary, and also sufficient, to require that one junction point of $\mathfrak{T}_{\mathrm{i}}$ be grounded, for all $i$. 
Assuming the calculation of $y$, by inversion of $z$, to be possible without too great difficulty, the final quantity required for the calculation of $A, B, \cdots, H$ and hence of $i^{\prime}, i^{\prime \prime}$ and $V$ is $y^{-1}$. In $y$ we have a two-term matrix the first term of which we haveassumed to be diagonalized in blocks and so invertible without too great difficulty; but, not having $y$ itself so diagonalized, our problem is to find a way to take advantage of the fact that one of its terms has this property in order to accomplish the inversion.

Bückner's formula for the inversion of $\left(B+K y K_{\mathrm{T}}\right)$, where $K$ is an $n \times t, t \leq n$, matrix of rank $t$ and $y$ is a $t \times t$ invertible matrix, is

$$
\left(B+K y K_{\mathrm{T}}\right)^{-1}=B^{-1}-B^{-1} K\left(z+K_{\mathrm{T}} B^{-1} K\right)^{-1} K_{\mathrm{T}} B^{-1} .
$$

This is a labor-saving substitute for any ordinary method of inversion of a two-termed matrix of this kind when $t \ll n$ and is then most valuable when $n$ is too great for any ordinary method of inversion to be economical. But its virtue diminishes to zero as $t \rightarrow n$.

3. A numerical case. We consider now what is about the simplest example that exhibits the various circumstances that aid or hinder the numerical inversion of a CauerRouth matrix. We consider a system of three grounded networks, $\mathfrak{N}_{1}, \mathfrak{N}_{2}, \mathfrak{N}_{3}$, inter-

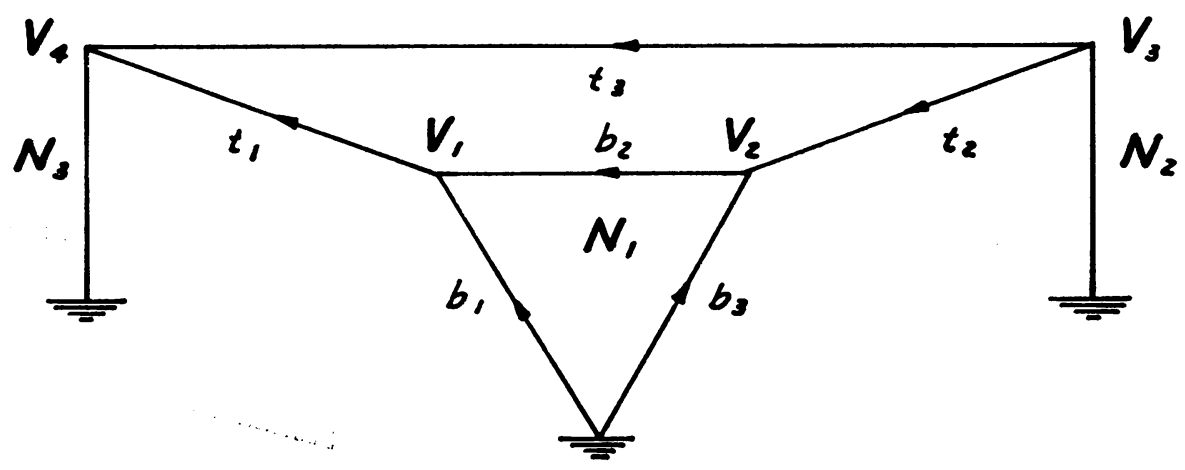

FIG. 1.

connected by three tie-lines, the network branch and tie-line impedances being purely resistive and purely diagonal:

$$
\begin{aligned}
& Z=[1,2,1 ; 1 ; 1), \quad z=[1,1,1) .
\end{aligned}
$$

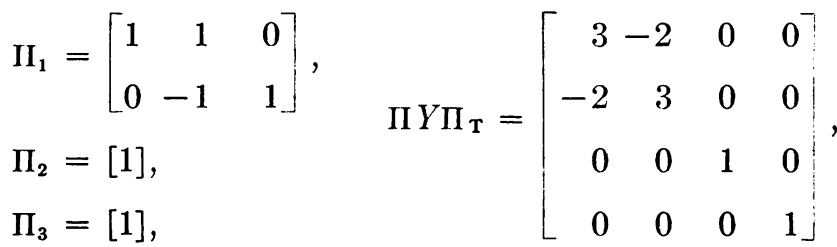

$$
\begin{aligned}
& K=\left[\begin{array}{rrr}
-1 & 0 & 0 \\
0 & 1 & 0 \\
0 & -1 & -1 \\
-1 & 0 & 1
\end{array}\right] \quad K y K_{\mathrm{T}}=\left[\begin{array}{rrrr}
1 & 0 & 0 & -1 \\
0 & 1 & -1 & 0 \\
0 & -1 & 2 & -1 \\
-1 & 0 & -1 & 2
\end{array}\right], \quad Y=\left[\begin{array}{rrrr}
4 & -2 & 0 & -1 \\
-2 & 4 & -1 & 0 \\
0 & -1 & 3 & -1 \\
-1 & 0 & -1 & 3
\end{array}\right] \text {. }
\end{aligned}
$$

Inversion of $\mathcal{Y}$ in this one-term form by partitioning requires 36 multiplications and 34 
additions. For inversion by the two-term formula we have

$$
\begin{gathered}
K_{\mathrm{T}} B^{-1} K=\frac{1}{5}\left[\begin{array}{rrr}
8 & -2 & 5 \\
-2 & 8 & 5 \\
5 & 5 & 10
\end{array}\right], \quad\left(z+K_{\mathrm{T}} B^{-1} K\right)^{-1}=\frac{1}{69}\left[\begin{array}{rrrr}
34 & 11 & -15 \\
11 & 34 & -15 \\
-15 & -15 & 33
\end{array}\right], \\
B^{-1} K\left[z+K_{\mathrm{T}} B^{-1} K\right]^{-1} K_{\mathrm{T}} B^{-1}=\frac{1}{25} \cdot \frac{1}{69}\left[\begin{array}{rrrr}
310 & 265 & -250 & -325 \\
265 & -170 & -325 & -250 \\
-250 & -325 & 925 & -350 \\
-325 & -250 & -350 & 925
\end{array}\right], \\
B^{-1}=\frac{1}{25 \times 69}\left[\begin{array}{cccc}
1035 & 690 & 0 & 0 \\
690 & 1035 & 0 & 0 \\
0 & 0 & 1725 & 0 \\
0 & 0 & 0 & 1725
\end{array}\right], \quad z=\frac{1}{69}\left[\begin{array}{cccc}
29 & 17 & 10 & 13 \\
17 & 29 & 13 & 10 \\
10 & 13 & 32 & 14 \\
13 & 10 & 14 & 32
\end{array}\right]
\end{gathered}
$$

Inversion of $y$ by this method requires 88 multiplications and 47 additions.

4. Networks with activated nodes. By such I mean a network of two parts: (1) a principal part consisting of a connected network $\Re$ whose graph $\mathcal{G}$ has $V$ vertices one of which is a ground vertex, and (2) a subsidiary part consisting of a set of $k, 1 \leq k \leq V-1$ $=n$, active dipoles each grounded at one pole and connected to a node of $\mathscr{N}$ at the other, the $k$ dipoles being in one-to-one correspondence with $k$ nodes.

Given the set of e.m.f.s, $e$, in the branches of $\mathscr{T}$ and the set of e.m.f.s, $\epsilon$, in the branches of the subsidiary network, assumed to be directed to $\Re$, we want to know the branch currents, $i$, in the principal part and the currents ` in the subsidiary part. For simplicity, we assume that the first $k$ nodes of $\Re$ have this outside connection.

Let $J$ be an $n \times n$ identity matrix with all columns deleted except the first $k$. Kirchhoff's node condition is

$$
\Pi i+J_{\iota}=0 .
$$

The e.m.f. equation, with $z$ the branch-impedance matrix of the subsidiary network, is

$$
\left[\begin{array}{ccc}
Z & 0 & \Pi_{\mathbf{T}} \\
0 & z & J_{\mathbf{T}} \\
\Pi & J & 0
\end{array}\right]\left[\begin{array}{c}
i \\
\iota \\
V
\end{array}\right]=\left[\begin{array}{c}
e \\
\epsilon \\
0
\end{array}\right] .
$$

On the pattern of Sec. 2, the branch currents and node voltages are

$$
\begin{aligned}
i & =\left(Y-Y \Pi_{\mathrm{T}} z \Pi Y\right) e-Y \Pi_{\mathrm{T}} z J y \epsilon, \\
\iota & =-y J_{\mathrm{T}} z \Pi Y e+\left[y-y J_{\mathrm{T}} z J y\right] \epsilon, \\
V & =z \Pi Y e+z J y \epsilon, \\
\mathcal{Y} & =\left[\Pi Y \Pi_{\mathrm{T}}+J y J_{\mathrm{T}}\right]=z^{-1} ;
\end{aligned}
$$

we note that the impedances to $e$ when $\epsilon=0$ and to $\epsilon$ when $e=0$ are 


$$
\begin{aligned}
{\left[Y-Y \Pi_{\mathrm{T}} z \Pi Y\right]^{-1} } & =Z+\Pi_{\mathrm{T}}\left(J y J_{\mathrm{T}}\right)^{-1} \Pi, \\
{\left[y-y J_{\mathrm{T}} z J y\right]^{-1} } & =z+J_{\mathrm{T}}\left(\Pi Y \Pi_{\mathrm{T}}\right)^{-1} J
\end{aligned}
$$

respectively.

Of special interest is the case $z=0$. The governing equations are

$$
\begin{aligned}
Z i+\Pi_{\mathbf{T}} V & =e, \\
J_{\mathbf{T}} V & =\epsilon, \\
\Pi i+J_{\iota} & =0 .
\end{aligned}
$$

By simple manipulations, we find $V$ and $i$ in terms of $e$ and $\iota$ :

$$
\begin{aligned}
V & =\left(\Pi Y \Pi_{\mathrm{r}}\right)^{-1} \Pi Y e+\left(\Pi Y \Pi_{\mathrm{T}}\right)^{-1} J_{\iota}, \\
i & =Y e-Y \Pi_{\mathrm{T}} V .
\end{aligned}
$$

Because $\epsilon=J_{\mathrm{T}} V$ we get the equation

$$
\epsilon-J_{\mathrm{T}}\left(\Pi Y \Pi_{\mathrm{T}}\right)^{-1} \Pi Y e=J_{\mathrm{T}}\left(\Pi Y \Pi_{\mathrm{T}}\right)^{-1} J_{\iota}
$$

which tell us that when the left member is zero the subsidiary network has no effect on $\Re$. This being true for all values of $J$, we see that

$$
\left(\Pi Y \Pi_{\mathrm{T}}\right)^{-1} \Pi Y e
$$

is the Lagrangian vector force of constraint $V$.

Let $V=V_{0}$ and $i=i_{0}$ when $J=0$. Then

$$
\begin{aligned}
V_{0} & =\left(\Pi Y \Pi_{\mathrm{T}}\right)^{-1} \Pi Y e, \\
i_{0} & =\left(Y-Y \Pi_{\mathrm{T}}\left(\Pi Y \Pi_{\mathrm{T}}\right)^{-1} \Pi Y\right) e .
\end{aligned}
$$

The increase of the node voltages, $\delta V$, and the decrease in the currents in the branches of $\Re, \delta i$, in the case $J \neq 0$ are easily found to be

$$
\begin{aligned}
\delta V & =\left(\Pi Y \Pi_{\mathrm{T}}\right)^{-1} J \iota, \\
\delta i & =Y \Pi_{\mathrm{T}} \delta V,
\end{aligned}
$$

and so the e.m.f. equation may be written

$$
e-\Pi_{\mathrm{T}}\left(V_{0}+\delta V\right)=Z(i-\delta i) .
$$

Recalling Cauer's equations for $\mathfrak{N}$ with $J=0$,

$$
i_{0}=\Gamma i^{\prime}, \quad e^{\prime}=\Gamma_{\mathrm{T}} e, \quad e^{\prime}=\Gamma_{\mathrm{T}} Z \Gamma i^{\prime},
$$

and Veblen's identity, $\Pi \Gamma=0$, the following algebraic chart epitomizes the topologicaldynamical theory.

The gap at the bottom is closed when and only when $\delta V=0$ and the gap at the top is closed when and only when $e=0$. For both gaps to be closed at the same time we must have a network $\mathfrak{N}$ with no current injected at any node and no current flowing in any branch- indeed a reductio ad absurdum!

5. Relation to Diakoptics. When $e=0$, then $V=\left(\Pi Y \Pi_{\mathbf{T}}\right)^{-1} J_{\iota}$, as we have seen. Kron's problem, according to Bückner [4, p. 57] and, primarily, the "Diakoptic" problem, 


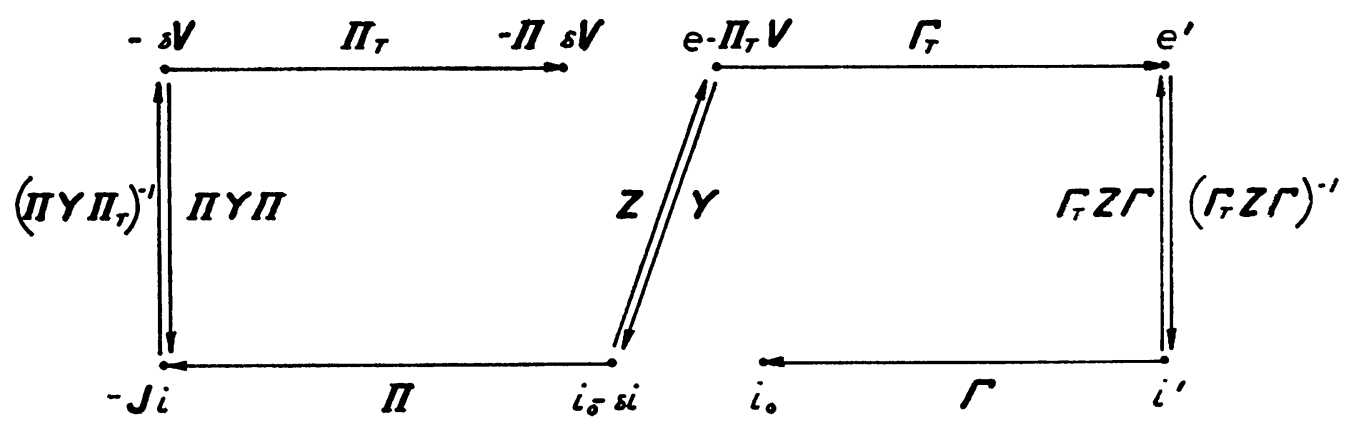

FIG. 2.

according to Braae, [5, Eq. 11.11] is to solve the equation

$$
Y^{\prime} V=\iota
$$

for $V$ when $Y^{\prime}$ is an invertable matrix imperfectly diagonalized in blocks.

Bückner's analysis of a problem of this kind is to consider the simultaneous system

$$
A x=f, \quad B x=g,
$$

where $A$ is a $n \times n$ matrix imperfectly diagonalized in blocks and $B$ is the same matrix with the spoiling elements replaced by zeros. Obviously $(A-B) x=f-g=f-B x$ and so

$$
B x=f-(A-B) x .
$$

Now $(A-B)$ is an $n \times n$ matrix of rank $r \leq n$ and, at this stage in his analysis, Bückner makes use of the fact that this difference can always be represented by the product of any $n \times r$ matrix $K$ of rank $r$ and any $r \times n$ matrix $L$ also of rank $r$.

It is not our purpose to reproduce Bückner's derivation of his formula, nor to attempt to follow Kron's thinking except to note that Bückner finds that, in effect, Kron has a "connection tensor", $C$, where he, Bückner, has $K$ and $C_{\mathrm{T}}$ where he has $L$. But the application of this tensor thinking to the tie-line problem has been thought to require an extensive defense in the literature. The explanation of this is that no directed-circuiton-branch incidence matrix $\Gamma$, or "connection tensor $C$ ", is appropriate to the diakoptic problem. On the other hand, our directed-branch-on-node incidence matrix $K$ of Sec. 3 is quite appropriate and reduces the tie-line problem to a question of the efficiency of Bückner's formula.

Addendum. In a private communication, J. L. Synge has supplied still another and most simple proof of the Bückner formula. Let $I_{n}$ and $I_{s}$ be identity matrices of $n$ and $t$ rows respectively and let $P$ and $Q$ be arbitrary except that $\left(I_{n}+Q P\right)^{-1}$ and $\left(I_{t}+P Q\right)^{-1}$ must exist. Then, obviously

$$
\begin{gathered}
P\left(I_{n}+Q P\right)=\left(I_{t}+P Q\right) P, \\
\therefore\left(I_{t}+P Q\right)^{-1} P\left(I_{n}+Q P\right)=P, \\
\therefore Q\left(I_{t}+P Q\right)^{-1} P\left(I_{n}+Q P\right)=Q P=\left(I_{n}+Q P\right)-I_{n}, \\
\therefore Q\left(I_{t}+P Q\right)^{-1} P=I_{n}-\left(I_{n}+Q P\right)^{-1}, \\
\therefore\left(I_{n}+Q P\right)^{-1}=I_{n}-Q\left(I_{t}+P Q\right)^{-1} P .
\end{gathered}
$$


This is the essence of the Bückner formula. To obtain the form used in Sec. 3, let

$$
P=y K_{\mathrm{T}}, \quad Q=B^{-1} K
$$

and then multiply through on the right by $B^{-1}$.

\section{REFERENCES}

[1] Edward John Routh, The advanced part of a treatise on the dynamics of a system of rigid bodies, being Part II of a treatise on the whole subject, 6th ed., Dover, New York, 1905

[2] Wilhelm Cauer, Untersuchungen über ein Problem, das drei positiv definite quadratische Formen mit Streckenkomplexen in Beziehung setzt: die Fassung der Arbeit durch Kürzung einer Abhandlung welche in 1928 der Redaktion vorgelegen hat, Math. Ann. 105, 86-132 (1931)

[3] W. H. Ingram and C. M. Cramlet, On the foundations of electrical network theory, J. Math. Phys., 23 134-155 (1944)

[4] H. F. Bückner, Die Anwendung elektronischer Rechenmaschinen in der Starkstromtechnik, Buchreihe Band 3, VDE-Verlag, Berlin, 1958.

[5] R. Braae, Matrix analysis for electrical engineers, Pitman, 1963. A proof of Bückner's formula for the case $L=K_{\mathrm{T}}$ is given on p. 47 and for the general case on p. 143; both proofs are simpler than Bückner's unpublisher proof.

150 Claremont Ave.,

New York 27, N. Y.
Box 353, Fire Island.

New York 11770 